\title{
Flat band in two-dimensional non-Hermitian optical lattices
}

\author{
S. M. Zhang and L. Jin (1)* \\ School of Physics, Nankai University, Tianjin 300071, China
}

(Received 11 July 2019; published 8 October 2019)

\begin{abstract}
We propose a method to generate a real-energy flat band in a two-dimensional (2D) non-Hermitian Lieb lattice. The coincidence of the flat-band eigenstate in both real and momentum spaces is essential for the proposed flat band, which is flexible at the appropriate match between the synthetic magnetic flux and non-Hermiticity. The proposed method is not limited to the 2D non-Hermitian Lieb lattice and is applied to the 2D non-Hermitian Tasaki's decorated square lattice, dice lattice, and kagome lattice. Our findings advance the flat-band engineering in $2 \mathrm{D}$ non-Hermitian optical lattices.
\end{abstract}

DOI: 10.1103/PhysRevA.100.043808

\section{INTRODUCTION}

The flat band is a dispersionless energy band with fixed energy [1]. It is independent of the system momentum and leads to unconventional Anderson localization [2,3] and AharonovBohm cages [4-8]. The superposition of flat-band eigenstates exhibits no diffractive dynamical behavior. Systems with flat bands have been a hot topic in the study of optics [9-24]. Synthetic magnetic flux in optical systems [25-30] enables the destructive interference resulting in a flat band. The eigenstates of the flat band are compact localized states (CLSs) [31-37] and the excitations of CLSs are confined to one or more unit cells.

Flat bands have been proposed and experimentally realized in a large number of two-dimensional (2D) lattices, including the 2D Lieb lattice [9-11], kagome lattice [18,19], honeycomb lattice [20], dice lattice [38], and others [32,39]. The Lieb lattice has a three-site unit cell; the chiral symmetry ensures energy spectra corresponding to the flat band with a zeroenergy and two symmetric dispersive bands, with the three energy spectra sharing a common Dirac point [40]. The chiral flat band in a generalized 3D Lieb lattice has also been verified to possess CLSs [31]. In addition, other 1D models such as the Lieb lattice [41], rhombic lattice [17,42,43], crossstitch lattice $[13,37,44]$, and triangular lattice [21] support flat bands.

Parity-time $(\mathcal{P} \mathcal{T})$ symmetry in non-Hermitian systems [45-48], in particular, a coupled $\mathcal{P} \mathcal{T}$-symmetric dimer system [49-54], has been widely investigated in various physical aspects [55-72], including coherent perfect absorption [59], unidirectional invisibility [60], wireless energy transfer [62], unidirectional propagation, and unidirectional lasing [71]. Topological photonic crystals with global non-Hermiticity have

\footnotetext{
*jinliang@nankai.edu.cn

Published by the American Physical Society under the terms of the Creative Commons Attribution 4.0 International license. Further distribution of this work must maintain attribution to the author(s) and the published article's title, journal citation, and DOI.
}

also been realized in experiments [73-80]. These provide an optical platform for the study of flat bands in non-Hermitian systems. In practice, flat bands appear in a large number of non-Hermitian systems [22,24,81-84]. Non-Hermitian systems, with the combination of a bipartite symmetry and frustration, support flat bands [22]. The flat band is maintained at the appropriate match between the synthetic magnetic flux and non-Hermiticity under destructive interference [81]. With the precondition of non-Hermitian particle-hole symmetry, photonic zero modes in a flat band can appear in the gain and loss modulated lattices [24]. In the quasi-1D cases, several types of lattices possess flat bands with the additional $\mathcal{P} \mathcal{T}$-symmetric gain and loss [83]; examples include the 1D Lieb lattice [82]. A flat band and light localization can be manipulated in a non-Hermitian $\mathcal{P} \mathcal{T}$-symmetric lattice [84].

In this study, we propose generation of flat bands in $2 \mathrm{D}$ non-Hermitian lattices. In the non-Hermitian generalization, a flat band in the Lieb lattice is maintained due to the destructive interference. The precondition for a tunable flat band is that the flat-band eigenstate in the momentum space is in accordance with that in the real space. The tunable flat-band energy, whose values are real numbers, is manipulated in terms of variation of system parameters, including the synthetic magnetic flux caused by a nonreciprocal phase factor and nonHermiticity. The position where a flat band appears in energy spectra is changeable and it intersects with one of the other two dispersive bands, except in the chiral symmetry condition. Notably, exceptional points (EPs) are doubly degenerate under the non-Hermitian condition. Compact localized states exist in such a model, and the time evolution of the initial excitation is stable as time increases. Furthermore, a simple popularization of the construction method of adjustable flat bands is also provided for other 2D lattice, including Tasaki's decorated square lattice, the dice lattice, and the kagome lattice.

The remainder of this paper is organized as follows. In Sec. II we introduce a 2D non-Hermitian Lieb lattice. The methods to achieve a tunable flat band and the energy band structure are introduced in Secs. III and IV, respectively. Compact localized states are depicted in Sec. V. Section VI introduces a generalization of the procedure to achieve a 


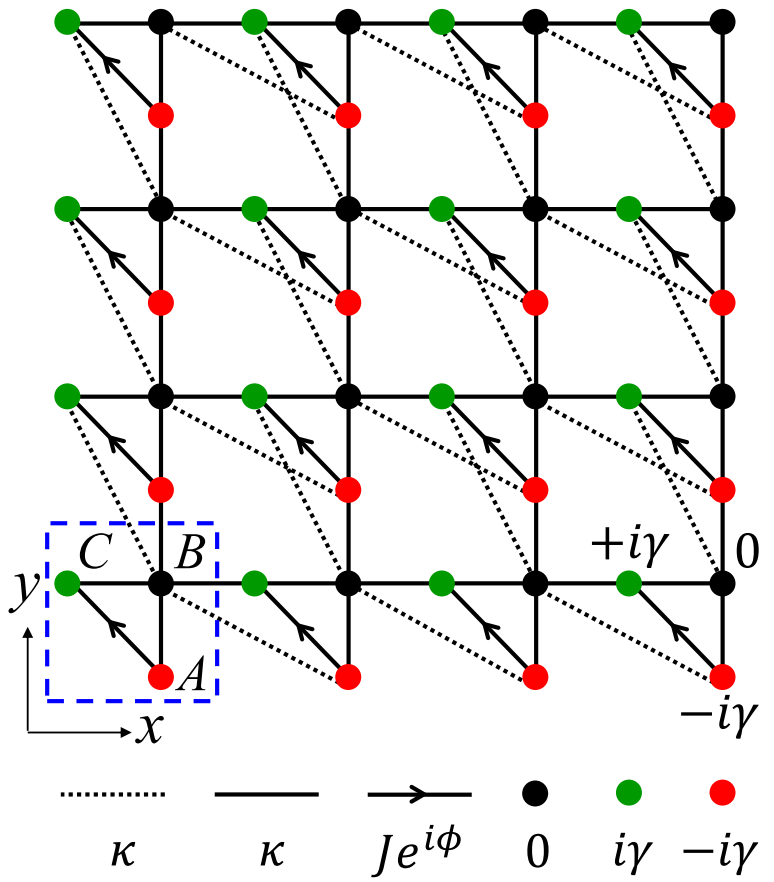

FIG. 1. Schematic of the two-dimensional Lieb lattice. The sites with gain (loss) are in green (red) and the sites that are passive are in black. The black arrow represents the nonreciprocal coupling, which is $J e^{i \phi}\left(J e^{-i \phi}\right)$ for photons tunneling along (against) the arrow. All the dotted and solid black lines indicate the reciprocal coupling $\kappa$. A unit cell involving sublattices $A, B$, and $C$ is exhibited by the blue dashed box.

tunable flat band for other 2D lattices. A summary is presented in Sec. VII.

\section{NON-HERMITIAN 2D LIEB LATTICE}

In the non-Hermitian generalization, $A, B$, and $C$ represent different types of sublattices; $A_{j}, B_{j}$, and $C_{j}$ constitute the $j$ th unit cell, depicted by the blue dashed box in Fig. 1. The nonHermitian Hamiltonian in the real space is given by

$$
\begin{aligned}
H= & \sum_{m, n}\left[\kappa b_{m, n}^{\dagger}\left(a_{m, n}+a_{m, n+1}+a_{m+1, n}\right)\right. \\
& \left.+\kappa b_{m, n}^{\dagger}\left(c_{m, n}+c_{m+1, n}+c_{m, n+1}\right)\right] \\
& +J e^{i \phi} c_{m, n}^{\dagger} a_{m, n}+\text { H.c. }+i \gamma\left(-a_{m, n}^{\dagger} a_{m, n}+c_{m, n}^{\dagger} c_{m, n}\right)
\end{aligned}
$$

where $a_{m, n}^{\dagger}$ and $a_{m, n}$ as well as $b_{m, n}^{\dagger}\left(c_{m, n}^{\dagger}\right)$ and $b_{m, n}\left(c_{m, n}\right)$ are the creation and annihilation operators, respectively. The parameter $\kappa$ represents the coupling strength between different sites, which can be deemed positive real numbers without loss of generality. The nonreciprocal coupling $J e^{ \pm i \phi}$ between lattices $A$ and $C$ means that the coupling amplitude is $J$, but its phase factor depends on the direction of photons tunneling. Photons tunneling from $A$ to $C$ will acquire a Peierls phase factor $e^{i \phi}$ [29,30]; in contrast, photons tunneling oppositely from $C$ to $A$ will acquire a Peierls phase factor $e^{-i \phi}$ [85]. The gain and loss rates are $\gamma$.

Under the periodic boundary condition, by taking the Fourier transformation $\rho_{m, n}^{\dagger}=(M N)^{-1 / 2} \sum_{\mathbf{k}} e^{-i\left(k_{x} m+k_{y} n\right)} \rho_{\mathbf{k}}^{\dagger}$
( $\rho=a, b, c)$, we obtain $H=\sum_{\mathbf{k}} H_{\mathbf{k}}$, where the wave vector $k_{x}=2 m \pi / M$ and $k_{y}=2 n \pi / N$, with $m$ and $n$ integers that range from 1 to $M$ and $N$, respectively. Here $H_{\mathbf{k}}$ is the Hamiltonian in the momentum space. Under the basis $\left\{a_{\mathbf{k}}^{\dagger}|\mathrm{vac}\rangle, b_{\mathbf{k}}^{\dagger}|\mathrm{vac}\rangle, c_{\mathbf{k}}^{\dagger}|\mathrm{vac}\rangle\right\}, H_{\mathbf{k}}$ is a $3 \times 3$ matrix in the form

$$
H_{\mathbf{k}}=\left(\begin{array}{ccc}
-i \gamma & \kappa \Lambda^{\dagger}(\mathbf{k}) & J e^{-i \phi} \\
\kappa \Lambda(\mathbf{k}) & 0 & \kappa \Lambda(\mathbf{k}) \\
J e^{i \phi} & \kappa \Lambda^{\dagger}(\mathbf{k}) & i \gamma
\end{array}\right)
$$

where $\Lambda(\mathbf{k})=1+e^{i k_{x}}+e^{i k_{y}}$. The $2 \mathrm{D}$ Lieb lattice maintains time-reversal symmetry $\mathcal{T} H_{\mathbf{k}} \mathcal{T}^{-1}=H_{-\mathbf{k}}$, where the timereversal operator $\mathcal{T}=R \mathcal{K}$ with the unitary operator $R$ and the complex conjugation operator $\mathcal{K}$. When $J=0$ or $\phi=$ $n \pi+\pi / 2(n \in \mathbb{Z})$, the Hamiltonian in the momentum space satisfies the chiral symmetry $C H_{\mathbf{k}} C^{-1}=-H_{\mathbf{k}}$. The unitary operator $R$ and the chiral operator $C$ are defined as

$$
R=\left(\begin{array}{lll}
0 & 0 & 1 \\
0 & 1 & 0 \\
1 & 0 & 0
\end{array}\right), \quad C=\left(\begin{array}{ccc}
0 & 0 & 1 \\
0 & -1 & 0 \\
1 & 0 & 0
\end{array}\right)
$$

From the determinant of the matrix $H_{\mathbf{k}}-E_{\mathbf{k}} I_{3 \times 3}\left(I_{3 \times 3}\right.$ is the $3 \times 3$ identity matrix), that is, $\operatorname{det}\left(H_{\mathbf{k}}-E_{\mathbf{k}} I_{3 \times 3}\right)=0$, we obtain a cubic equation of $E_{\mathbf{k}}$,

$$
E_{\mathbf{k}}^{3}-\left(2 \kappa^{2} s_{\mathbf{k}}+J^{2}-\gamma^{2}\right) E_{\mathbf{k}}-2 \kappa^{2} J s_{\mathbf{k}} \cos \phi=0,
$$

where $s_{\mathbf{k}}=2 \cos \left(k_{x}-k_{y}\right)+2\left(\cos k_{x}+\cos k_{y}\right)+3$. Notably, the parameter $s_{\mathbf{k}}$ is within the range of $s_{\mathbf{k}} \in[0,9]$. The energy bands can be analytically obtained from the solution of the cubic equation.

In the following section, we discuss the energy bands of the non-Hermitian Lieb lattice. We show that (i) a zero-energy flat band presents in the lattice with chiral symmetry and (ii) the flat band persists at an appropriate match between the nonreciprocal coupling and non-Hermiticity in the absence of chiral symmetry. We are not interested in the trivial situation $(\kappa=0)$, where all unit cells are isolated.

\section{FLAT BANDS}

Under chiral symmetry, the spectrum of the system is symmetric about zero energy. A three-band chiral symmetric system must have a zero-energy flat band and two dispersive bands with opposite energies. Equation (4) reduces to $E_{\mathbf{k}}\left(E_{\mathbf{k}}^{2}+\gamma^{2}-2 \kappa^{2} s_{\mathbf{k}}\right)=0$ for $J=0$ or $E_{\mathbf{k}}\left(E_{\mathbf{k}}^{2}+\gamma^{2}-J^{2}-\right.$ $\left.2 \kappa^{2} s_{\mathbf{k}}\right)=0$ for $\phi=n \pi+\pi / 2(n \in \mathbb{Z})$. Thus, a zero-energy flat band with $E_{\mathbf{k}}=0$ is protected by the chiral symmetry.

The original 2D Lieb lattice without non-Hermiticity and synthetic magnetic flux supports a zero-energy flat band [9], that is, only the nearest-neighbor couplings [solid black lines in Fig. 1(a)] present in $H_{\mathbf{k}}$. The three energy bands are given by 0 and $\pm \kappa \sqrt{2 \cos k_{x}+2 \cos k_{y}+4}$. The zero-energy band is independent of the momentum and thus it is a dispersionless flat band. The eigenstate in the momentum space for the 
zero-energy flat band as a result of destructive interference is

$$
f_{\mathbf{k}}=\left(-\frac{1+e^{i k_{x}}}{1+e^{i k_{y}}}, 0,1\right)^{\mathrm{T}} \text {. }
$$

The sublattice $B$ only vertically couples with sublattice $A$ and only horizontally couples with sublattice $C$; the difference leads to the momentum-dependent amplitude in $f_{\mathbf{k}}$.

In the extended Lieb lattice, the destructive interference is maintained, consequently leading to the formation of a flat band at the destructive interference. This will be discussed in detail. The sites in sublattice $B$ are unoccupied at the destructive interference; to keep them unoccupied, first, additional long-range couplings $\kappa$ are applied, as indicated by the black dotted lines in Fig. 1. The destructive interference at sublattice $B$ and the flat band are unchanged; however, the form of the eigenstates $f_{\mathbf{k}}$ in the Bloch Hamiltonian $H_{\mathbf{k}}$ alters under the additional long-range couplings $\kappa$. The corresponding zeroenergy flat-band eigenstate changes to

$$
f_{\mathbf{k}}=(-1,0,1)^{\top} \text {. }
$$

Under the influence of the additional couplings $\kappa$, the zeroenergy eigenstate in the momentum space $(-1,0,1)^{\top}$ coincides with the eigenstate for each unit cell in the real space $\left\{A_{j}, B_{j}, C_{j}\right\}=\{-1,0,1\}$. It is worth noting the significance of the formation of a destructive interference at sublattice $B$, while sublattices $A$ and $C$ have wave functions -1 and 1 , respectively. This feature is critical for the existence of the flat band in the non-Hermitian extension, because sublattices $A$ and $C$ can be engineered to keep the eigenstate and destructive interference unchanged. In this way, the flat-band energy is tunable under non-Hermitian engineering.

Maintenance of the destructive interference is the precondition for the formation of a flat band, and the wave functions of three sublattices in the case of flat bands ought not to be changed, although some extra elements are supplemented by adding extra gain and loss $\pm i \gamma$, respectively, to sublattices $A$ and $C$ associated with a nonreciprocal phase factor in the coupling $J e^{ \pm i \phi}$ between them. Here $A_{j}$ and $C_{j}$ in each unit cell constitute a $\mathcal{P} \mathcal{T}$-symmetric dimer [45-50,55,56,58,72] and -1 and 1 constitute an eigenstate of the dimer at an appropriate condition [81]. By $H_{\mathbf{k}}$ acting on the flat-band wave function $f_{\mathbf{k}}=\left(\psi_{A}, \psi_{B}, \psi_{C}\right)^{\top}$, the Schrödinger equations $H_{\mathbf{k}}\left(\psi_{A}, \psi_{B}, \psi_{C}\right)^{\top}=E_{\mathbf{k}}\left(\psi_{A}, \psi_{B}, \psi_{C}\right)^{\top}$ can be written as

$$
\begin{aligned}
& E_{\mathbf{k}} \psi_{A}=\kappa \psi_{B}\left(e^{-i k_{x}}+e^{-i k_{y}}+1\right)-i \gamma \psi_{A}+J e^{-i \phi} \psi_{C}, \\
& E_{\mathbf{k}} \psi_{B}=\kappa \psi_{A}\left(e^{i k_{x}}+e^{i k_{y}}+1\right)+\kappa \psi_{C}\left(e^{i k_{x}}+e^{i k_{y}}+1\right), \\
& E_{\mathbf{k}} \psi_{C}=\kappa \psi_{B}\left(e^{-i k_{x}}+e^{-i k_{y}}+1\right)+i \gamma \psi_{C}+J e^{i \phi} \psi_{A} .
\end{aligned}
$$

After substituting $\left(\psi_{A}, \psi_{B}, \psi_{C}\right)^{\top}=(-1,0,1)^{\top} \quad$ into Schrödinger equations, we obtain two relations $i \gamma+J e^{-i \phi}=$ $-E_{\mathbf{k}}$ and $i \gamma-J e^{i \phi}=E_{\mathbf{k}}$, which provide the flat-band condition

$$
\gamma=J \sin \phi
$$

as well as the flat-band energy

$$
E_{\mathbf{k}}=-J \cos \phi \text {. }
$$

It is evident that the adjustable flat-band energy is real. Obviously, the real energy of the flat band is tunable by varying the coupling $J$ or the phase $\phi$ at the appropriate match, independent of the reciprocal intersublattice couplings $\kappa$. The unchanged eigenstate implies the maintenance of the flat band. To summarize, the momentum-independent eigenstate is essential and beneficial for the flat band in the non-Hermitian extended Lieb lattice. To achieve the flat band in the 2D non-Hermitian Lieb lattice, two steps are elucidated. First, through adding extra couplings, a momentum-independent flat-band eigenstate is obtained, which is the key role that the long-range couplings $\kappa$ play in the destructive interference. Second, the gain and loss $\pm i \gamma$ and nonreciprocal couplings $J e^{ \pm i \phi}$ are added at an appropriate match; the eigenstate is unchanged, thereby rendering the flat-band energy tunable.

\section{ENERGY BANDS}

All the points of intersection of the energy spectra contain degenerate points (DPs) and EPs. The DPs and EPs are found from the discriminant $\Delta=27 \kappa^{4} J^{2} s_{\mathbf{k}}^{2} \cos ^{2} \phi-$ $\left(2 \kappa^{2} s_{\mathbf{k}}+J^{2} \cos ^{2} \phi\right)^{3}$ of the cubic equation [Eq. (4)] about the energy bands under the flat-band condition. Here $\gamma=J=0$ yields the Hermitian Lieb lattice. The cubic equation of $E_{\mathbf{k}}$ is simplified to $E_{\mathbf{k}}\left(E_{\mathbf{k}}^{2}-2 \kappa^{2} s_{\mathbf{k}}\right)=0$. A zero-energy flat band exists. In this situation, the discriminant $\Delta=0$ is satisfied only when $s_{\mathbf{k}}=0$; the three bands intersect at the zero energy and the intersection points are DPs. Otherwise, the band intersections are EPs in the non-Hermitian case $(\gamma \neq 0)$. The discriminant $\Delta=0$ yields the condition

$$
J^{2} \cos ^{2} \phi-\kappa^{2} s_{\mathbf{k}}=0,
$$

provided that $s_{\mathbf{k}} \in[0,9]$. We emphasize that $\phi=n \pi+\pi / 2$ $(n \in \mathbb{Z})$ corresponds to the case that the system possesses the chiral symmetry. The chiral symmetry protects the existence of a zero-energy flat band, and it is not necessary to satisfy Eq. (10) to form the zero-energy flat band. Although all three energies are zeros at band intersections, only two eigenstates coalesce and the two intersections are two-state coalescence (EP2) regardless of the couplings $J$ and $\kappa$. For $\phi \neq n \pi+\pi / 2$, the flat band intersects a dispersive band. The EPs appear as an isolated EP or EP ring. A single isolated EP appears at $k_{x}=$ $k_{y}=0$ when the flat band is at the bottom or top of the three bands, there are two isolated EPs or DPs in the presence of chiral symmetry, and the intersections are single or double EP rings in other cases. The EPs may disappear if $J^{2} \cos ^{2} \phi / \kappa^{2}$ exceeds the value of $s_{\mathbf{k}}$. Since $s_{\mathbf{k}} \in[0,9], J^{2} \cos ^{2} \phi>9 \kappa^{2}$ corresponds to the situation that EPs are absent; consequently, the three bands are separated.

We analyze the spectra of the non-Hermitian Lieb lattice. The spectrum of the original Hermitian Lieb lattice is presented in Fig. 2(a). The addition of long-range couplings $\kappa$ to the traditional Lieb lattice does not change the energy of the flat band. The Lieb lattice is still Hermitian and the corresponding spectrum is presented in Fig. 2(b). The zeroenergy points in the spectrum are two DPs. The positions of the DPs in the Brillouin zone (BZ) are fixed and independent of $\kappa$ and $\phi$. The condition of Eq. (12) yields the existence of EPs. The appearance of EP2 in our discussion with the nonexistence of three-state coalescence indicates that the equality 
(a)

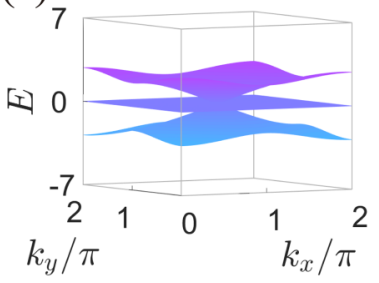

(c)

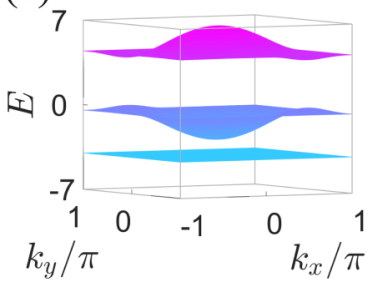

(e)

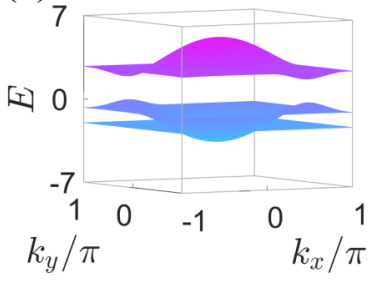

(b)

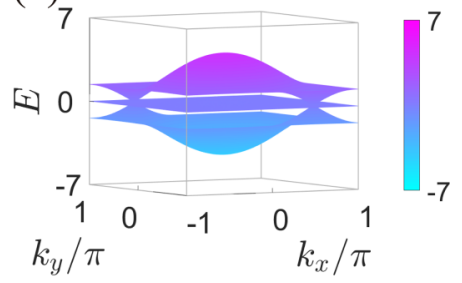

(d)

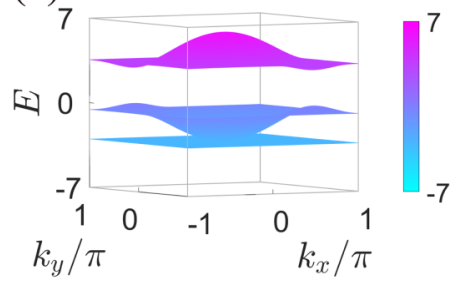

(f)

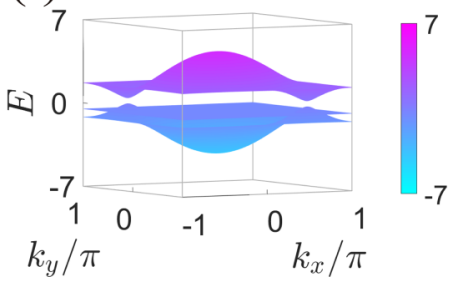

FIG. 2. Energy spectra of (a) the original version of the Hermitian Lieb lattice and (b) the Hermitian lattice with additional longrange couplings $\kappa$. Also shown are non-Hermitian energy spectra with a flat band at (c) $J=8$, (d) $J=6$, (e) $J=4$, and (f) $J=1$; the parameter $\phi=\pi / 3$ is fixed. For all the spectra, the other coupling $\kappa=1$ is set.

is satisfied. Figures 2(c)-2(f) represent energy spectra for a certain constant $\phi \in[0, \pi / 2)$. Figure 2(c) is a representative spectrum for the separable energy bands without any EP. In Fig. 2(d), the flat band and the lower dispersive band share a common point in the BZ, which is an isolated EP2 at $k_{x}=k_{y}=0$; this requires an appropriate match between $\kappa$ and $J$, that is, $J^{2} \cos ^{2} \phi=9 \kappa^{2}$. If $J / \kappa$ decreases, the flat-band energy increases and the band intersection changes from a single EP to an EP ring [Fig. 2(e)] and then to two EP rings [Fig. 2(f)]. After that, the band intersection ends up with two DPs at $J=0$. When $\phi \in(\pi / 2, \pi]$, the flat band intersects with the upper band. The relative positions of the flat band and the dispersive bands are similar to those in the case of $\phi \in[0, \pi / 2)$. As $J / \kappa$ increases from 0 , the flat-band energy increases to a positive value and the band intersections change from two EP rings to a single EP ring and finally to a single isolated $\mathrm{EP}\left(k_{x}=k_{y}=0\right)$ at $J^{2} \cos ^{2} \phi=9 \kappa^{2}$. Subsequently, increasing $J / \kappa$ results in the separable band structure with the flat band being the upper band.

\section{COMPACT LOCALIZED STATES}

The CLSs can be found at the flat-band condition (10). The destructive interference at sublattice $B$ indicates that the wave functions of sublattices $A$ and $C$ satisfy $\psi_{A_{m, n}}+\psi_{C_{m, n}}=0$ for the $n$th column with the $m$ th line unit cell. The Schrödinger equations of the sites in the $n$th column with the $m$ th line unit

cell inside the non-Hermitian Lieb lattice under the periodic boundary condition are given by [86]

$$
\begin{gathered}
i \dot{\psi}_{A_{m, n}}=\kappa\left(\psi_{B_{m, n}}+\psi_{B_{m, n-1}}+\psi_{B_{m-1, n}}\right)+J e^{-i \phi} \psi_{C_{m, n}}-i \gamma \psi_{A_{m, n}} \\
i \dot{\psi}_{B_{m, n}}= \\
\quad \kappa\left(\psi_{A_{m, n}}+\psi_{C_{m, n}}\right)+\kappa\left(\psi_{A_{m+1, n}}+\psi_{C_{m+1, n}}\right) \\
+\kappa\left(\psi_{A_{m, n+1}}+\psi_{C_{m, n+1}}\right)
\end{gathered}
$$

$i \dot{\psi}_{C_{m, n}}=\kappa\left(\psi_{B_{m, n}}+\psi_{B_{m, n-1}}+\psi_{B_{m-1, n}}\right)+J e^{i \phi} \psi_{A_{m, n}}+i \gamma \psi_{C_{m, n}}$.

At the steady state, the Schrödinger equations reduce to

$$
E \psi_{A_{m, n}}=\kappa\left(\psi_{B_{m, n}}+\psi_{B_{m, n-1}}+\psi_{B_{m-1, n}}\right)-i \gamma \psi_{A_{m, n}}+J e^{-i \phi} \psi_{C_{m, n}},
$$

$$
\begin{aligned}
& E \psi_{B_{m, n}}=\kappa\left(\psi_{A_{m, n}}+\psi_{A_{m+1, n}}+\psi_{A_{m, n+1}}\right) \\
& +\kappa\left(\psi_{C_{m, n}}+\psi_{C_{m+1, n}}+\psi_{C_{m, n+1}}\right) \text {, } \\
& E \psi_{C_{m, n}}=\kappa\left(\psi_{B_{m, n}}+\psi_{B_{m, n-1}}+\psi_{B_{m-1, n}}\right)+i \gamma \psi_{C_{m, n}}+J e^{i \phi} \psi_{A_{m, n}} .
\end{aligned}
$$

The solution is $\left\{\psi_{A_{m, n}}, \psi_{B_{m, n}}, \psi_{C_{m, n}}\right\}=\{-1,0,1\}$, with the amplitudes of the other unit cells being $\{0,0,0\}$. This indicates that the CLS of the Schrödinger equations in the real space is in accordance with the eigenstate solutions of the Schrödinger equations in the momentum space.

The CLS has a different form in the situation where the Lieb lattice possesses chiral symmetry at $\phi=\pi / 2$, but $\gamma \neq$ $J$. Under chiral symmetry, $J$ does not have to satisfy $\gamma=$ $J \sin (\pi / 2)$. The corresponding CLSs of the zero-energy flat band at $\gamma \neq J$ localize in three unit cells. The representative Schrödinger equations for the three unit cells at the bottom left are given by

$$
\begin{gathered}
i \dot{\psi}_{A_{1}}=\kappa \psi_{B_{1}}-i \gamma \psi_{A_{1}}+J e^{-i \phi} \psi_{C_{1}}, \\
i \dot{\psi}_{B_{1}}=\kappa\left(\psi_{A_{1}}+\psi_{C_{1}}+\psi_{A_{2}}+\psi_{C_{2}}+\psi_{A_{3}}+\psi_{C_{3}}\right), \\
i \dot{\psi}_{C_{1}}=i \gamma \psi_{C_{1}}+\kappa \psi_{B_{1}}+J e^{i \phi} \psi_{A_{1}} .
\end{gathered}
$$

Substituting $\phi=\pi / 2$ into the Schrödinger equations, the steady-state solutions of the non-normalized CLSs are

$$
\begin{gathered}
\psi_{A_{1}}=\psi_{A_{2}}=\psi_{A_{3}}=-1, \\
\psi_{B_{1}}=i(J-\gamma) / \kappa, \psi_{B_{2}}=\psi_{B_{3}}=0 \\
\psi_{C_{1}}=\psi_{C_{2}}=\psi_{C_{3}}=1 .
\end{gathered}
$$

Notably, similar structures of CLSs appear at other corners and inside the non-Hermitian Lieb lattice.

The time evolution of CLSs is obtained as

$$
|\Phi(t)\rangle=e^{-i H t}|\Phi(0)\rangle .
$$



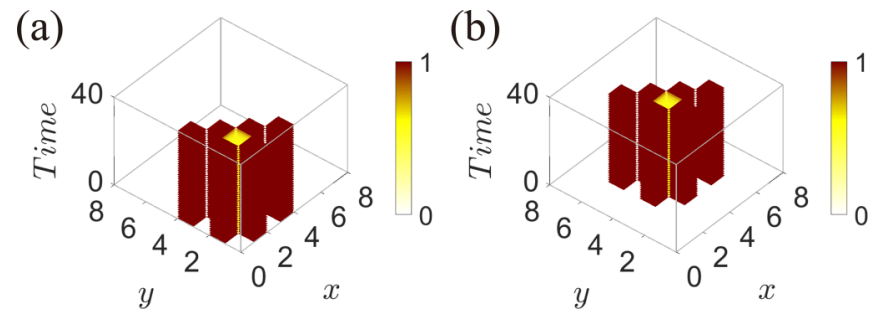

FIG. 3. Time evolution $|\langle\Phi(t) \mid \Phi(t)\rangle|^{2}$ of CLSs (a) at the lattice boundary and (b) inside the lattice. The unit of time is $1 / \kappa$. The initial excitations are in the form of Eqs. (22)-(24). The Lieb lattice is chiral symmetric under the system parameters $J=1, \kappa=1, \phi=\pi / 2$, and $\gamma=1 / 4$.

The initial excitations $|\Phi(0)\rangle$ are chosen as when the CLSs are at the lattice boundary and inside the lattice. The numerical simulations of the dynamics are shown in Fig. 3, where the excitation intensity $|\langle\Phi(t) \mid \Phi(t)\rangle|^{2}$ is depicted. In the simulation, we choose eight sites in both the $x$ and $y$ directions. The initial excitation located at the edge of the lattice in Fig. 3(a) is provided by Eqs. (22)-(24) with $|\Phi(0)\rangle=(-1,3 i / 4,1,-1,0,1,-1,0,1)^{\top}$. In Fig. 3(b), the initial excitation is located inside the lattice. The nonexcited zero intensities in both numerical simulation diagrams are displayed in white. We notice that the profiles of the initial excitations are unchanged in the whole dynamical process, being confined and diffractionless. The time evolution dynamics verifies that the CLSs are localized in three unit cells in the non-Hermitian Lieb lattice under chiral symmetry $\phi=$ $n \pi+\pi / 2(n \in \mathbb{Z})$, but $\gamma \neq J \sin \phi$.

\section{TASAKI'S DECORATED SQUARE LATTICE AND THE DICE LATTICE}

A flat band can be realized in other 2D non-Hermitian lattices by employing the approach introduced in Sec. III. In this section, as examples, we apply the two steps to propose a tunable flat band in the Tasaki's decorated square lattice, the dice lattice, and the kagome lattice. In the first step, we propose a 2D lattice possessing a momentum-independent flat band for the lattice in the momentum space; in the second step, we apply additional terms involving non-Hermitian elements to manipulate the flat-band energy without changing the eigenstate of the flat band.

The Tasaki's decorated square lattice is schematically illustrated in Fig. 4(a), which is a 2D Lieb lattice with extra long-range couplings of four nearest-neighbor sites for sublattice $B$. Applying the same approaches elucidated for the aforementioned Lieb lattice, a momentum-independent eigenstate is achieved for the flat band that is similar to that for the Lieb lattice. In addition, the gain and loss associated with the nonreciprocal couplings added in the Tasaki's decorated square lattice similar to the 2D non-Hermitian Lieb lattice maintain the flat band, and the flat-band energy and eigenstate are identical to those of the non-Hermitian Lieb lattice previously discussed in Sec. II. The tunable flat-band energy $-J \cos \phi$ is depicted in Fig. 4(d). (a)

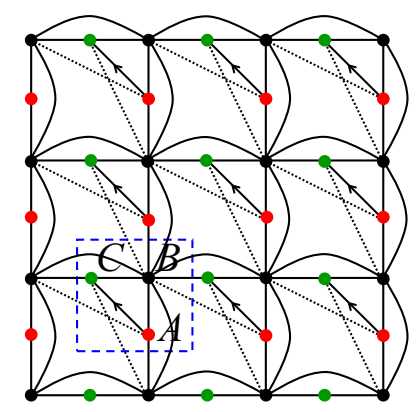

(d)

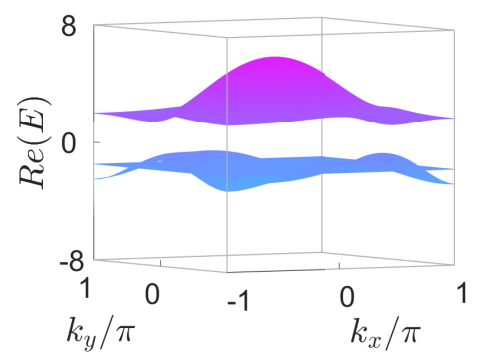

(b)

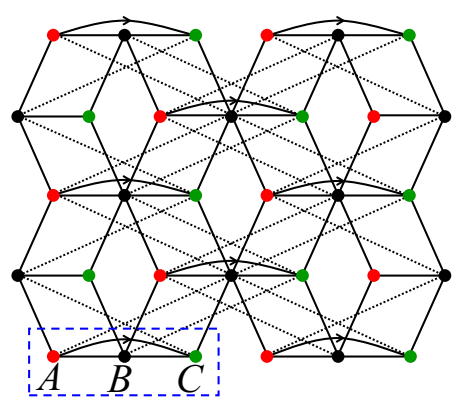

(e)

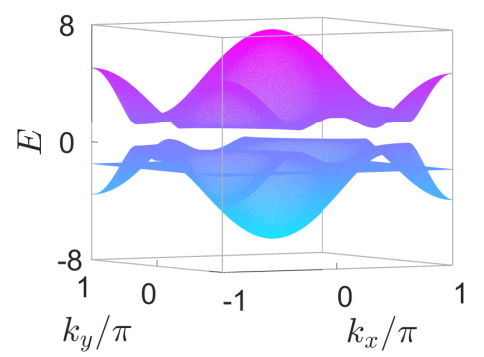

(c)

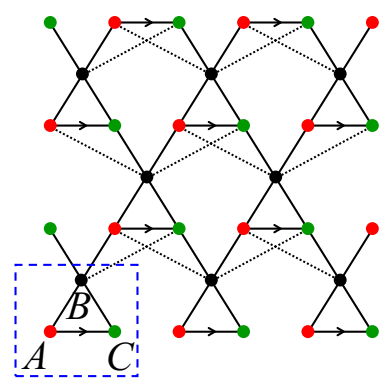

(f)

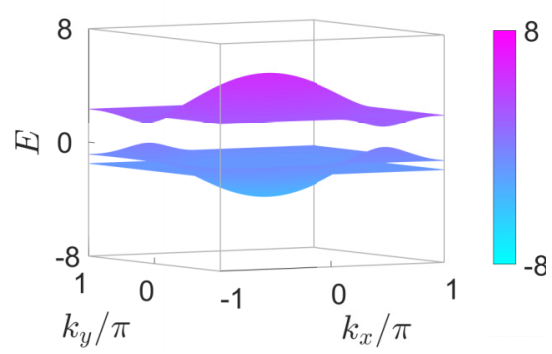

FIG. 4. Schematics of the 2D non-Hermitian (a) Tasaki's decorated square lattice, (b) dice lattice, and (c) kagome lattice and their corresponding real part of the energy spectra in (d), (e), and (f), respectively. The unit cells of all the lattices, indicated in the blue dashed boxes, contain three sites. The legends are identical to those shown in Fig. 1. All the band energies in the spectra are real numbers except for the two dispersive bands in (d). In (d)-(f) the parameters are $\phi=\pi / 3, \kappa=1$, and $J=3$. 
Furthermore, the non-Hermitian dice lattice depicted in Fig. 4(b) supports a tunable flat band. The configuration of coupling between sublattices $B$ and $C$ is not symmetric with respect to that between sublattices $B$ and $A$, which results in the momentum-dependent eigenstate for the zero-energy flat band of the dice lattice. This indicates that the addition of extra couplings is required in the original dice lattice to obtain the momentum-independent eigenstate of the flat band. The additional couplings are represented by the black dotted lines. Then the couplings between sublattices $B$ and $A$ are equal to those between sublattices $B$ and $C$. In the second step, with the appropriate match between the added nonreciprocal coupling and the non-Hermitian gain and loss, the flat band is formed at the destructive interference. The corresponding flat-band energy in Fig. 4(e) is identical to that in Fig. 4(d).

In Fig. 4(c), the method to achieve tunable flat bands in non-Hermitian systems is applied for the kagome lattice. For the original kagome model, the flat band is the lowest energy band with all couplings being positive; the flat-band energy is nonzero, which differs from that in the Lieb, Tasaki's decorated square, and dice lattices. The couplings between sublattices $A$ and $C$ are no longer zero in this case, thus making the first step to form the momentum-independent eigenstate of the flat band different; eliminating the couplings $\kappa$ between sublattices $A$ and $C$ solves the problem. The second step in achieving the flat band is similar to that for the aforementioned 2D lattices. The spectrum is depicted in Fig. 4(f); the flat-band energy is identical to those in Figs. 4(d) and 4(e).

\section{SUMMARY}

We have proposed a method to realize a flat band in a 2D non-Hermitian Lieb lattice, whose energy is flexible by the interplay of the non-Hermiticity and synthetic magnetic flux. We proposed two steps to achieve a 2D non-Hermitian lattice that supports a flat band. In the first step, we obtained the momentum-independent eigenstate of the flat band for the Bloch Hamiltonian, which corresponds to CLSs in its unit cell. In the second step, a nonreciprocal coupling and non-Hermitian gain and loss were introduced at appropriate matches to maintain the destructive interference. The flatband energy is tunable for the different matches between the coupling and the non-Hermitian gain and loss. Furthermore, we applied our approach to other 2D lattices. The proposed non-Hermitian Tasaki's decorated square lattice and the dice lattice were demonstrated to support tunable flat bands. We provided an alternative perspective on the generation of the flat bands, and our findings are applicable to a vast range of 2D Hermitian lattices and beyond for achieving flat bands in non-Hermitian lattices.

\section{ACKNOWLEDGMENTS}

This work was supported by National Natural Science Foundation of China (Grants No. 11975128 and No. 11605094) and the Fundamental Research Funds for the Central Universities, Nankai University (Grants No. 63191522 and No. 63191738).
[1] J. Vidal, R. Mosseri, and B. Douçot, Phys. Rev. Lett. 81, 5888 (1998).

[2] P. W. Anderson, Phys. Rev. 109, 1492 (1958).

[3] J. T. Chalker, T. S. Pickles, and P. Shukla, Phys. Rev. B 82, 104209 (2010)

[4] J. Vidal, B. Doucot, R. Mosseri, and P. Butaud, Phys. Rev. Lett. 85, 3906 (2000).

[5] S. Longhi, Opt. Lett. 39, 5892 (2014).

[6] J. Vidal, P. Butaud, B. Douçot, and R. Mosseri, Phys. Rev. B 64, 155306 (2001)

[7] S. Mukherjee, M. Di Liberto, P. Öhberg, R. R. Thomson, and N. Goldman, Phys. Rev. Lett. 121, 075502 (2018).

[8] M. Kremer, I. Petrides, E. Meyer, M. Heinrich, O. Zilberberg, and A. Szameit, arXiv:1805.05209.

[9] D. Guzmán-Silva, C. Mejía-Cortés, M. A. Bandres, M. C. Rechtsman, S. Weimann, S. Nolte, M. Segev, A. Szameit, and R. A. Vicencio, New J. Phys. 16, 063061 (2014).

[10] R. A. Vicencio, C. Cantillano, L. Morales-Inostroza, B. Real, C. Mejía-Cortés, S. Weimann, A. Szameit, and M. I. Molina, Phys. Rev. Lett. 114, 245503 (2015).

[11] S. Mukherjee, A. Spracklen, D. Choudhury, N. Goldman, P. Öhberg, E. Andersson, and R. R. Thomson, Phys. Rev. Lett. 114, 245504 (2015).

[12] A. Julku, S. Peotta, T. I. Vanhala, D.-H. Kim, and P. Törmä, Phys. Rev. Lett. 117, 045303 (2016).

[13] J. D. Bodyfelt, D. Leykam, C. Danieli, X. Yu, and S. Flach, Phys. Rev. Lett. 113, 236403 (2014).
[14] D. Leykam, S. Flach, O. Bahat-Treidel, and A. S. Desyatnikov, Phys. Rev. B 88, 224203 (2013).

[15] L. Ge, Ann. Phys. (Berlin) 529, 1600182 (2017).

[16] V. Apaja, M. Hyrkás, and M. Manninen, Phys. Rev. A 82, 041402(R) (2010).

[17] A. Bermudez, T. Schaetz, and D. Porras, Phys. Rev. Lett. 107, 150501 (2011).

[18] N. Masumoto, N. Y. Kim, T. Byrnes, K. Kusudo, A. Löffler, S. Höfling, A. Forchel, and Y. Yamamoto, New J. Phys. 14, 065002 (2012)

[19] G.-W. Chern, C.-C. Chien, and M. Di Ventra, Phys. Rev. A 90, 013609 (2014).

[20] C. Wu, D. Bergman, L. Balents, and S. Das Sarma, Phys. Rev. Lett. 99, 070401 (2007).

[21] P. Shukla, Phys. Rev. B 98, 054206 (2018).

[22] D. Leykam, S. Flach, and Y. D. Chong, Phys. Rev. B 96, 064305 (2017).

[23] L. Ge, Photon. Res. 6, A10 (2018).

[24] B. Qi, L. Zhang, and L. Ge, Phys. Rev. Lett. 120, 093901 (2018).

[25] Z. Yu and S. Fan, Nat. Photonics 3, 91 (2009).

[26] K. Fang, Z. Yu, and S. Fan, Phys. Rev. Lett. 108, 153901 (2012).

[27] E. Li, B. J. Eggleton, K. Fang, and S. Fan, Nat. Commun. 5, 3225 (2014).

[28] P. Roushan et al., Nat. Phys. 13, 146 (2017).

[29] M. Hafezi, Int. J. Mod. Phys. B 28, 1441002 (2014).

[30] M. Hafezi, Phys. Rev. Lett. 112, 210405 (2014). 
[31] A. Ramachandran, A. Andreanov, and S. Flach, Phys. Rev. B 96, 161104(R) (2017).

[32] D. Leykam, A. Andreanov, and S. Flach, Adv. Phys. X 3, 1473052 (2018).

[33] C. W. Hsu, B. Zhen, A. D. Stone, J. D. Joannopoulos, and M. Soljačić, Nat. Rev. Mater. 1, 16048 (2016).

[34] Y.-X. Xiao, G. Ma, Z.-Q. Zhang, and C. T. Chan, Phys. Rev. Lett. 118, 166803 (2017).

[35] W. Maimaiti, A. Andreanov, H. C. Park, O. Gendelman, and S. Flach, Phys. Rev. B 95, 115135 (2017).

[36] S. Flach, D. Leykam, J. D. Bodyfelt, P. Matthies, and A. S. Desyatnikov, Europhys. Lett. 105, 30001 (2014).

[37] C. Gneiting, Z. Li, and F. Nori, Phys. Rev. B 98, 134203 (2018).

[38] A. R. Kolovsky, A. Ramachandran, and S. Flach, Phys. Rev. B 97, 045120 (2018).

[39] X. Li, E. Zhao, and W. V. Liu, Nat. Commun. 4, 1523 (2013).

[40] E. H. Lieb, Phys. Rev. Lett. 62, 1201 (1989).

[41] F. Baboux, L. Ge, T. Jacqmin, M. Biondi, E. Galopin, A. Lemaître, L. Le Gratiet, I. Sagnes, S. Schmidt, H. E. Türeci, A. Amo, and J. Bloch, Phys. Rev. Lett. 116, 066402 (2016).

[42] R. Khomeriki and S. Flach, Phys. Rev. Lett. 116, 245301 (2016).

[43] S. Mukherjee and R. R. Thomson, Opt. Lett. 40, 5443 (2015); 42, 2243 (2017).

[44] M. Tovmasyan, S. Peotta, L. Liang, P. Törmä, and S. D. Huber, Phys. Rev. B 98, 134513 (2018).

[45] C. M. Bender and S. Boettcher, Phys. Rev. Lett. 80, 5243 (1998).

[46] A. Ruschhaupt, F. Delgado, and J. G. Muga, J. Phys. A: Math. Gen. 38, L171 (2005).

[47] S. Klaiman, U. Günther, and N. Moiseyev, Phys. Rev. Lett. 101, 080402 (2008).

[48] R. El-Ganainy, K. G. Makris, D. N. Christodoulides, and Z. H. Musslimani, Opt. Lett. 32, 2632 (2007); K. G. Makris, R. ElGanainy, D. N. Christodoulides, and Z. H. Musslimani, Phys. Rev. Lett. 100, 103904 (2008); Z. H. Musslimani, K. G. Makris, R. El-Ganainy, and D. N. Christodoulides, ibid. 100, 030402 (2008).

[49] A. Guo, G. J. Salamo, D. Duchesne, R. Morandotti, M. VolatierRavat, V. Aimez, G. A. Siviloglou, and D. N. Christodoulides, Phys. Rev. Lett. 103, 093902 (2009).

[50] C. E. Rüter, K. G. Makris, R. El-Ganainy, D. N. Christodoulides, M. Segev, and D. Kip, Nat. Phys. 6, 192 (2010).

[51] B. Peng, S. K. Özdemir, F. Lei, F. Monifi, M. Gianfreda, G. L. Long, S. Fan, F. Nori, C. M. Bender, and L. Yang, Nat. Phys. 10, 394 (2014).

[52] L. Chang, X. Jiang, S. Hua, C. Yang, J. Wen, L. Jiang, G. Li, G. Wang, and M. Xiao, Nat. Photon. 8, 524 (2014).

[53] H. Hodaei, M.-A. Miri, M. Heinrich, D. N. Christodoulides, and M. Khajavikhan, Science 346, 975 (2014).

[54] W. Chen, S. K. Ozdemir, G. Zhao, J. Wiersig, and L. Yang, Nature (London) 548, 192 (2017).

[55] L. Jin and Z. Song, Phys. Rev. A 80, 052107 (2009); 81, 032109 (2010).

[56] Y. N. Joglekar, D. Scott, M. Babbey, and A. Saxena, Phys. Rev. A 82, 030103(R) (2010).

[57] R. Driben and B. A. Malomed, Opt. Lett. 36, 4323 (2011).

[58] X. Luo, J. Huang, H. Zhong, X. Qin, Q. Xie, Y. S. Kivshar, and C. Lee, Phys. Rev. Lett. 110, 243902 (2013).
[59] Y. D. Chong, L. Ge, H. Cao, and A. D. Stone, Phys. Rev. Lett. 105, 053901 (2010); W. Wan, Y. Chong, L. Ge, H. Noh, A. D. Stone, and H. Cao, Science 331, 889 (2011); Y. Sun, W. Tan, H.Q. Li, J. Li, and H. Chen, Phys. Rev. Lett. 112, 143903 (2014).

[60] L. Feng, Y.-L. Xu, W. S. Fegadolli, M.-H. Lu, J. E. B. Oliveira, V. R. Almeida, Y.-F. Chen, and A. Scherer, Nat. Mater. 12, 108 (2013).

[61] H. Hodaei, A. U. Hassan, S. Wittek, H. Garcia-Gracia, R. ElGanainy, D. N. Christodoulides, and M. Khajavikhan, Nature (London) 548, 187 (2017).

[62] S. Assawaworrarit, X. Yu, and S. Fan, Nature (London) 546, 387 (2017).

[63] L. Feng, Z. J. Wong, R.-M. Ma, Y. Wang, and X. Zhang, Science 346, 972 (2014).

[64] X. Zhu, H. Ramezani, C. Shi, J. Zhu, and X. Zhang, Phys. Rev. X 4, 031042 (2014).

[65] R. Fleury, D. Sounas, and A. Alù, Nat. Commun. 6, 5905 (2015).

[66] Z. Zhang, Y. Zhang, J. Sheng, L. Yang, M.-A. Miri, D. N. Christodoulides, B. He, Y. Zhang, and M. Xiao, Phys. Rev. Lett. 117, 123601 (2016).

[67] A. Cerjan, A. Raman, and S. Fan, Phys. Rev. Lett. 116, 203902 (2016); A. Cerjan and S. Fan, ibid. 118, 253902 (2017).

[68] L. Feng, R. El-Ganainy, and L. Ge, Nat. Photon. 11, 752 (2017).

[69] Y. Kominis, T. Bountis, and S. Flach, Phys. Rev. A 95, 063832 (2017).

[70] R. El-Ganainy, K. G. Makris, M. Khajavikhan, Z. H. Musslimani, S. Rotter, and D. N. Christodoulides, Nat. Phys. 14, 11 (2018).

[71] L. Jin and Z. Song, Phys. Rev. Lett. 121, 073901 (2018).

[72] H. Schomerus, Phys. Rev. Lett. 104, 233601 (2010); S. Malzard, C. Poli, and H. Schomerus, ibid. 115, 200402 (2015).

[73] A. Szameit, M. C. Rechtsman, O. Bahat-Treidel, and M. Segev, Phys. Rev. A 84, 021806(R) (2011).

[74] J. M. Zeuner, M. C. Rechtsman, Y. Plotnik, Y. Lumer, S. Nolte, M. S. Rudner, M. Segev, and A. Szameit, Phys. Rev. Lett. 115, 040402 (2015).

[75] K. Ding, G. Ma, M. Xiao, Z. Q. Zhang, and C. T. Chan, Phys. Rev. X 6, 021007 (2016).

[76] D. L. Sounas and A. Alù, Nat. Photon. 11, 774 (2017).

[77] S. Longhi, Y. Kominis, and V. Kovanis, Europhys. Lett 122, 14004 (2018).

[78] H. Xu, D. Mason, L. Jiang, and J. G. E. Harris, Nature (London) 537, 80 (2016).

[79] B. Peng, S. K. Özdemir, M. Liertzer, W. Chen, J. Kramer, H. Yılmaz, J. Wiersig, S. Rotter, and L. Yang, Proc. Natl. Acad. Sci. U.S.A. 113, 6845 (2016).

[80] G. Harari, M. A. Bandres, Y. Lumer, M. C. Rechtsman, Y. D. Chong, M. Khajavikhan, D. N. Christodoulides, and M. Segev, Science 359, eaar4003 (2018); M. A. Bandres, S. Wittek, G. Harari, M. Parto, J. Ren, M. Segev, D. Christodoulides, and M. Khajavikhan, ibid. 359, eaar4005 (2018).

[81] L. Jin, Phys. Rev. A 99, 033810 (2019).

[82] L. Ge, Phys. Rev. A 92, 052103 (2015).

[83] M. I. Molina, Phys. Rev. A 92, 063813 (2015).

[84] H. Ramezani, Phys. Rev. A 96, 011802(R) (2017).

[85] L. Jin and Z. Song, Phys. Rev. A 93, 062110 (2016); L. Jin, ibid. 97, 012121 (2018); 97, 033840 (2018).

[86] Z. Chen, J. Liu, S. Fu, Y. Li, and B. A. Malomed, Opt. Express 22, 29679 (2014). 